\title{
Comparison of Ultrasound and Lenstar Biometry Methods in Determining Axial Length and Refractive Error after Cataract Surgery
}

\author{
Fatemeh Eslami $^{1}$, Mehdi Alizadeh ${ }^{1, *}$, Fatemeh Kosari ${ }^{2}$ \\ ${ }^{1}$ Assistant Professor, Department of Ophthalmology, School of Medicine, Hamadan University of Medical Sciences, Hamadan, \\ Iran \\ ${ }^{2}$ General Practitioner, Hamadan University of Medical Sciences, Hamadan, Iran
}

* Corresponding Author: Mehdi Alizadeh, Department of Ophthalmology, School of Medicine, Hamadan University of Medical Sciences, Hamadan, Iran. Email: mahaliz@yahoo.com

Received: 28.06.2018

Accepted: 10.11 .2018

\section{How to Cite this Article:} Eslami F, Alizadeh M, Kosari F. Comparison of Ultrasound and Lenstar Biometry Methods in Determining Axial Length and Refractive Error after Cataract Surgery. Avicenna J Clin Med. 2018; 25(3): 165-169. DOI: 10.21859/ajcm.25.3.165

\section{Abstract}

Background and Objective: Ocular biometry refers to the measurement of the axial length of the eye and thickness of the intraocular structures. This process is routinely performed for all patients before cataract surgery. The accuracy of the biometric data is directly associated with the refractive status after cataract surgery. Currently, two methods of biometry, namely ultrasound and optical biometry, are used to calculate the intraocular lens power. The purpose of this study was to compare the Lenstar and ultrasound biometry methods in determining the axis length and refractive error after cataract surgery.

Materials and Methods: This descriptive cross-sectional study was conducted on 372 patients with cataract. The study population was randomly divided into two groups of Lenstar and ultrasound based on the biometry methods they received. A total of 17 patients were excluded from the study due to having mature and dense cataract. Therefore, the study was continued with 355 patients, 186 and 169 cases of whom were managed with ultrasound and Lenstar biometry methods, respectively. Postoperative refractive errors were evaluated in each patient 4-6 weeks after the surgery. The two groups were compared in terms of the mean absolute refractive error using paired sample t-test.

Results: The mean absolute refractive errors before the surgery were obtained as $2.38 \pm 3.93$ and $2.19 \pm 2.68$ diopter in the ultrasound and Lenstar groups, respectively, which were not significantly different between the two groups $(\mathrm{P}=0.2)$. The mean axial lengths of the eye were $23.10 \pm 1.19$ and $23.34 \pm 1.17 \mathrm{~mm}$ in the ultrasound and Lenstar groups, respectively. The two groups showed no significant difference in this regard $(\mathrm{P}=0.96)$. After the operation, the mean absolute refractive errors in the ultrasound and Lenstar groups were $0.56 \pm 0.45$ and $0.51 \pm 0.38$ diopter, respectively. This variable was not significantly different between the two groups after the surgery $(\mathrm{P}=0.11)$.

Conclusion: The findings of this study revealed no significant difference between the ultrasound and Lenstar biometry methods in terms of determining the axis length and mean absolute refractive error after cataract surgery.

Keywords: Biometry, Cataract, Refractive Error 


\title{
مقايسه دو روش بيومترى التراسوند (Ultrasound) و لنستار (Lenstar) در تعيين طول محور جشم و ميزان عيب انكسارى پسى از عمل جراحى كاتاراكت
}

\author{
فاطمه اسلامى'، مهرى عليزاده ا“*، فاطمه كوثرى \\ ' استاديار، كروه جشميزشكى، دانشكده يزشكى، دانشكاه علوم بز شكى همدان، همدان، ايران

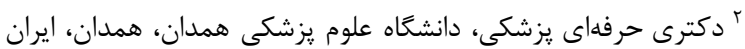

* نويسنده مسئول: مهدى عليزاده، گَروه جشمريزشكى، دانشكده يزشكى، دانشعاه علوم يزشكى همدان، همدان، ايران. ايميل:mahaliz@yahoo.com

\begin{tabular}{|c|c|}
\hline 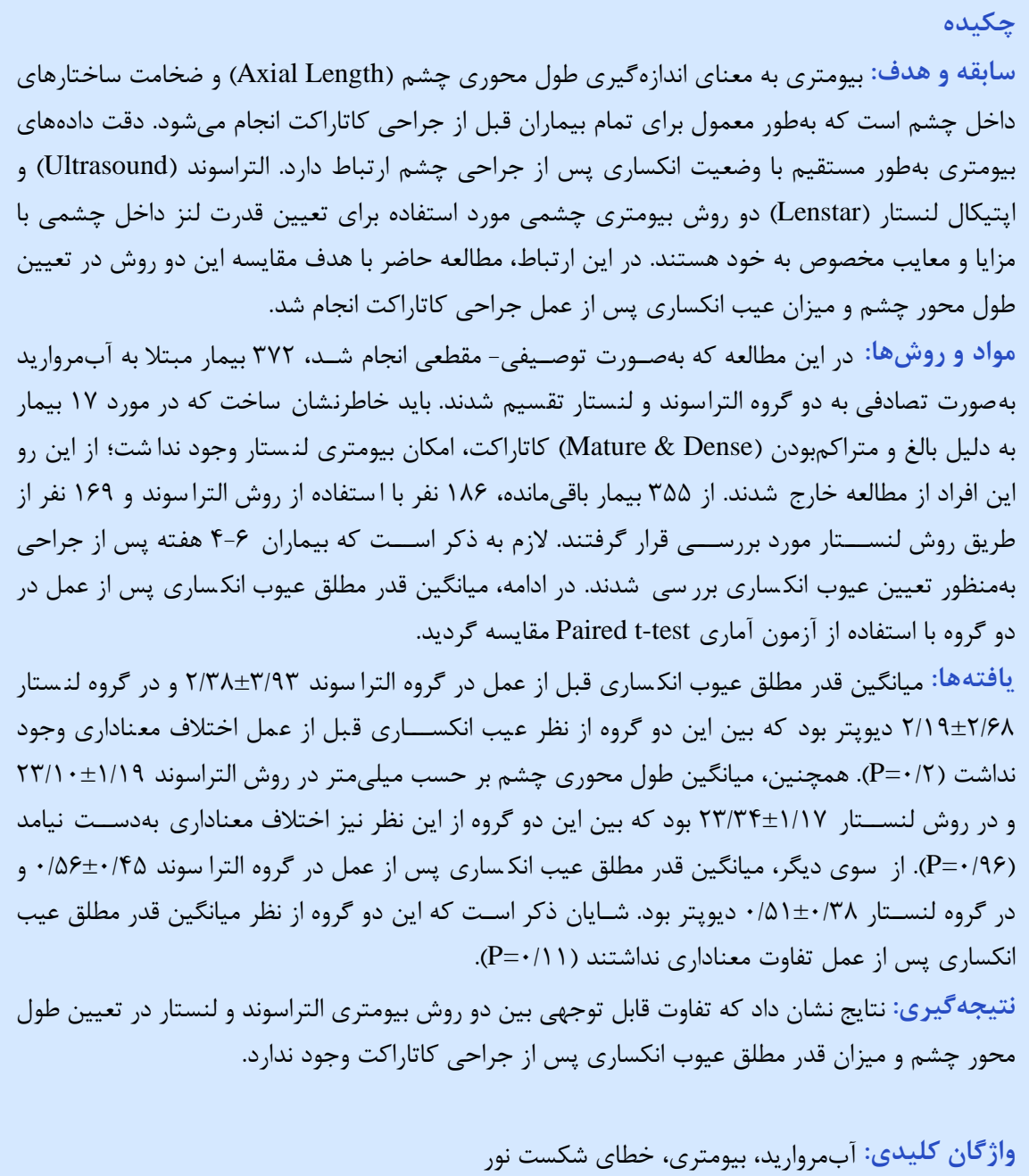 & 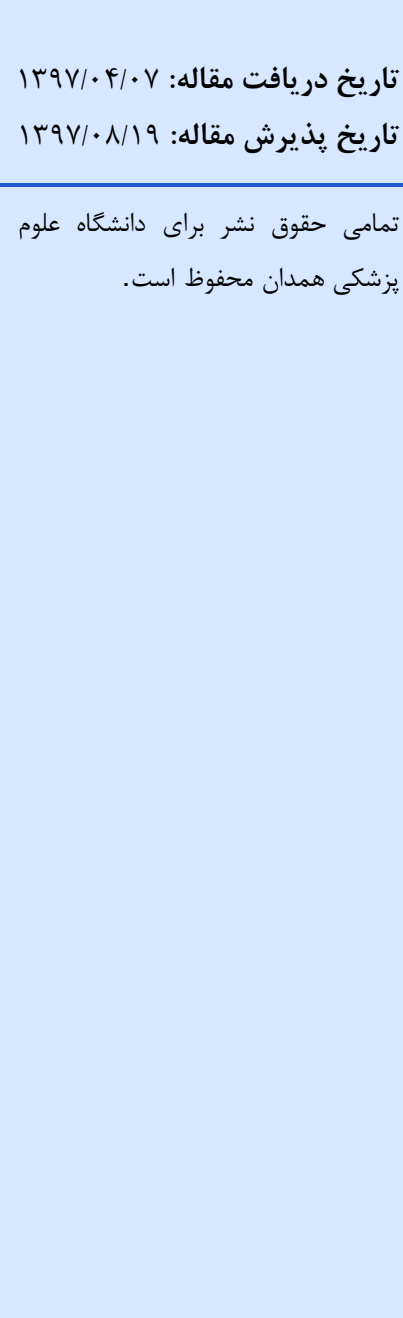 \\
\hline
\end{tabular}

اينكه دلايل تغذيهاى و عفونى ايجادكننده كورى كاملاً ريشهكن

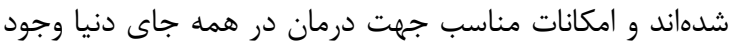

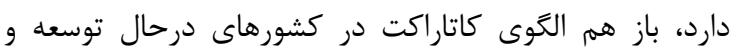

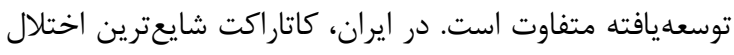

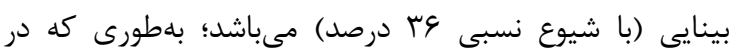

طبق برآورد سازمان جهانى بهداشت، آبمرواريد شايعترين

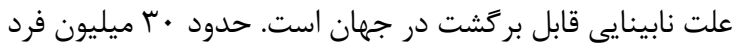

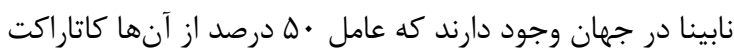
است [1]]. بر مبناى مطالعات انجامشده، تعداد افراد مبتلا به داند

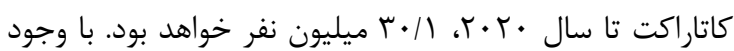


بيمار مبتلا به كاتاراكت كه در سال ه9

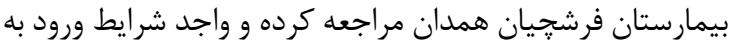

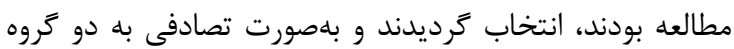

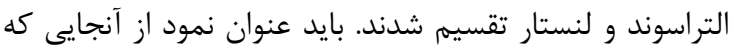
براى VI بيمار به دليل بالغ و متراكمبودن كاتاراكت، امكان

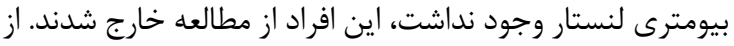

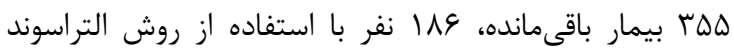

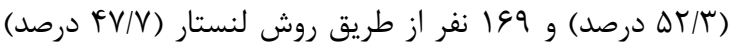

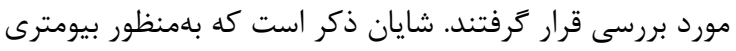

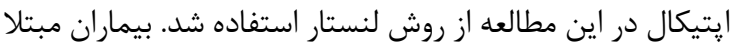

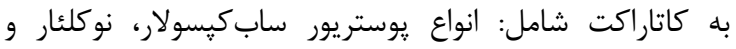

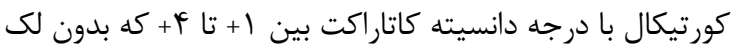

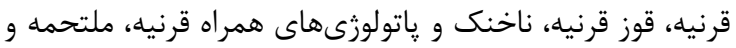

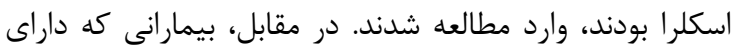

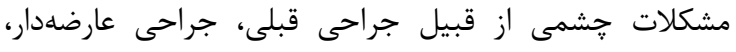

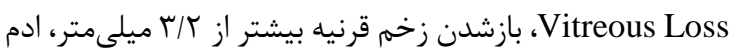

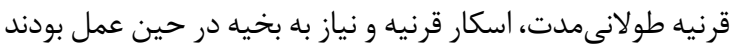

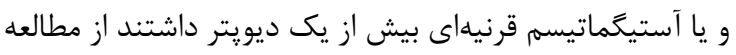

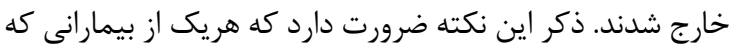

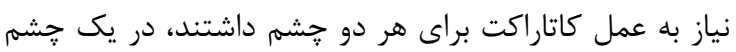

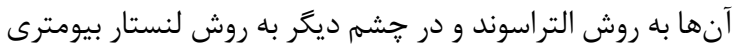

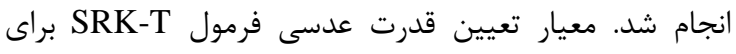
بيومترى در نظر گرفته شد و لنز مورد استفاده براى بيماران از شركت ALCON تهيه گرديد. در هفته Y-F يس از جراحى، بيماران بلمنظور تعيين عيوب

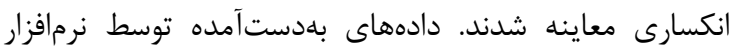

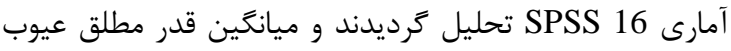
انكسارى (Refractive Error MARE: Mean Absolute) بعد از عمل دو گروه با استفاده از آزمون Paired t-test مقايسه

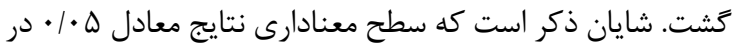
نظر گرفته شد.

إفته ه.

ميانگين سن بيماران در گروه التراسوند 99 سال و در گروه

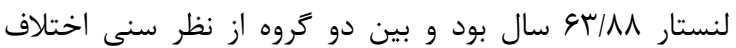

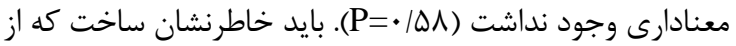

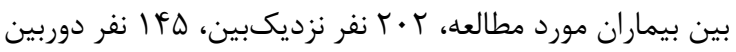
و 1 نفر بدون عيوب انكسارى بودند.

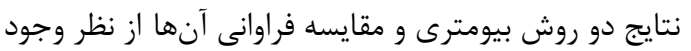

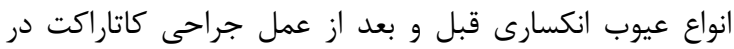
جدول ا مشاهده ميىشود.

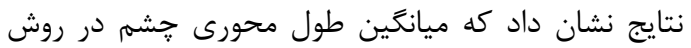

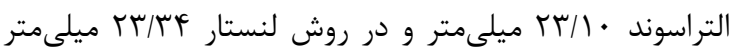
است و بين دو گروه از اين نظر اختلاف معنادارى وجود ندارد.
يك ينجم از جمعيت بالاى · F سال، ابتلا به كاتاراكت مشاهده

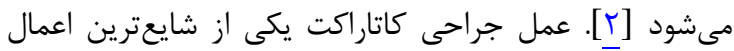

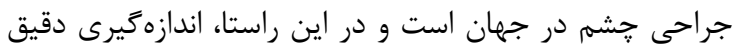

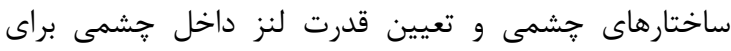

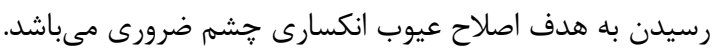

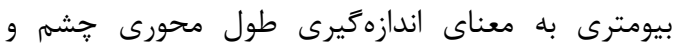

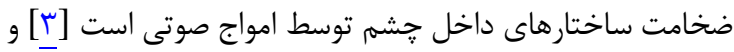

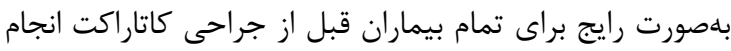

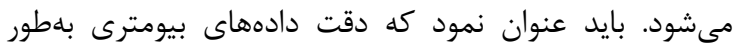

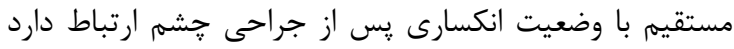

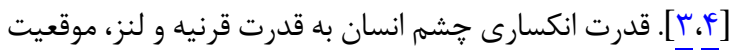

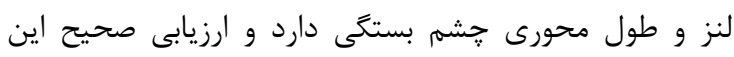
پارامترها بهمنظور دستيافتن به نتيجه انكسارى صحيح يس از از

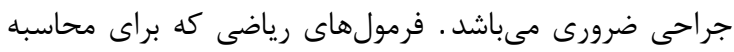

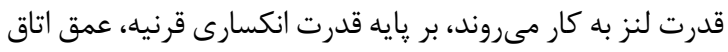

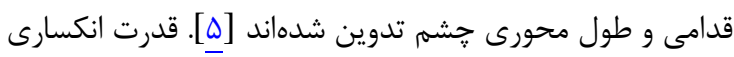

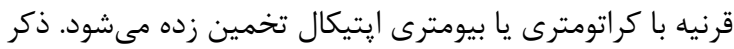

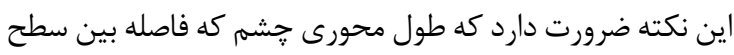

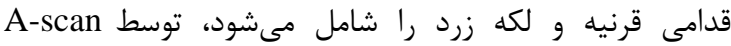

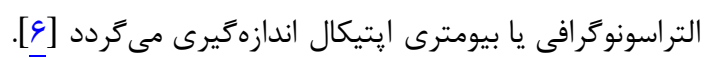
بيومترى التراسوند بر يايه تابش امواج التراسوند است؛ لئ

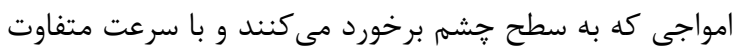

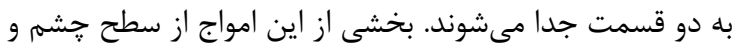

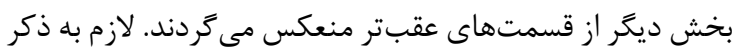

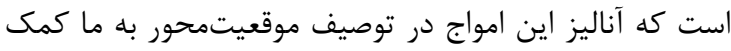
مى كند [V] بلهور رايج دو وسيله بيومترى إيتيكال در بازار وجود دارد.

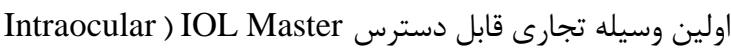
Carl Zeiss Meditec) (Lenses Haag-Streit AG) Lenstar LS 900 معرفى شده است. IOL Master از ليزر ديودى استفاده مى كند

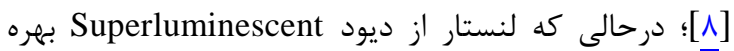

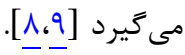

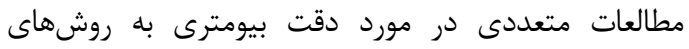

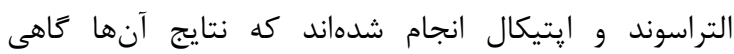

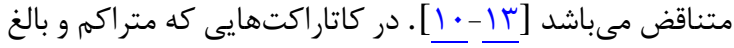
هستند، بيومترى به روش إتيكال قابل (Mature \& Dense)

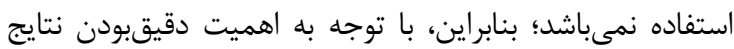

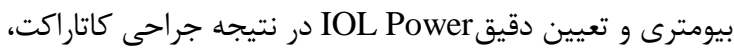

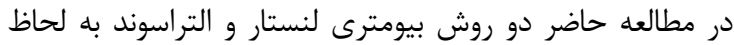
دقت محاسبه قدرت لنز داخل جشمى با يكديخر مقايسه شدند.

مواد و روشها

در اين مطالعه كه بهصورت توصيفى - مقطعى انجام شد، MVT 
مطلق عيوب انكسارى قبل از عمل و بعد از آن در هر دو روش

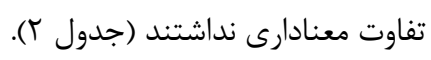

بين ميانكين قدرت لنز داخل جشمى نيز در دو روش بيومترى

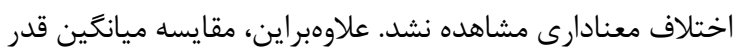

جدول ا: مقايسه دو روش بيومترى التراسوند و لنستار بر حسب انواع عيوب انكسارى قبل و بعد از عمل جراحى كاتاراكت

\begin{tabular}{|c|c|c|c|c|}
\hline \multicolumn{2}{|c|}{ بعد از جراحى (درصد) } & \multicolumn{2}{|c|}{ قبل از جراحى (درصد) } & \multirow{2}{*}{ عيوب انكسارى (تعداد) } \\
\hline ل ل ل ل & التراسوند & 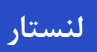 & التراسوند & \\
\hline$r 4 / .9$ & $r q 1 . r$ & SI/AT & $\Delta T / F T$ & نزديكبين (ץ+r نفر) \\
\hline$\Delta \Delta / \cdot \varphi$ & $\Delta 1 / \cdot V$ & $r \varepsilon / 9$ & $f \Delta / f$. & دوربين (هاfl نفر) \\
\hline N/AV & $11 / \pi 9$ & T/RG & r//9 & بدون عيب (^ نفر) \\
\hline
\end{tabular}

جدول ب: مقايسه دو روش التراسوند و لنستار بر حسب ميانكَين دادههاى بيومترى و ميانكَين قدر مطلق عيوب انكسارى قبل و بعد از عمل جراحى كاتاراكت

\begin{tabular}{|c|c|c|c|}
\hline سطح معنادارى & (لنستار & (ع التراسوند & متغير ها \\
\hline .199 & $r r / T F \pm I / l V$ & $r r / 1 \cdot \pm 1 / 19$ & طول محورى جشم (ميلى متر ) \\
\hline . AT & $19 / 1 r \pm r / \Delta 9$ & $19 / 49 \pm r / 49$ & قدرت لنز داخل جشمى (ديويتر) \\
\hline$\cdot \pi \cdot$ & $r / 19 \pm r / 9 \wedge$ & $r / r \Lambda \pm r / q r$ & قدر مطلق عيب انكسارى قبل از جراحى \\
\hline$\cdot 111$ & $\cdot|\Delta| \pm \cdot / \mu \wedge$ & $\cdot \mid \Delta s \pm \cdot / \& \Delta$ & قدر مطلق عيب انكسارى بعد از جراحى \\
\hline
\end{tabular}

التراسوند مقايسه شد. در اين مطالعه اختلاف ميانگين طول

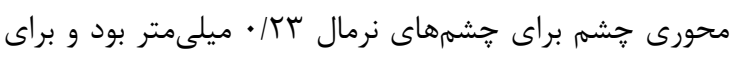

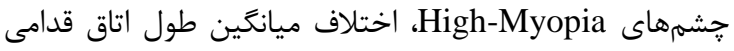
معادل ف/ • ميلىمتر محاسبه شد كه اين اختلاف هم براى

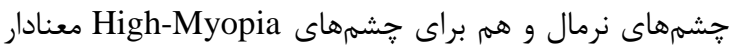

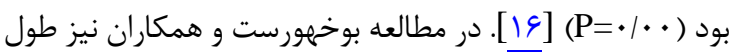

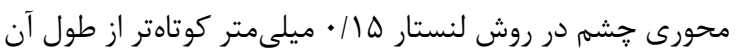

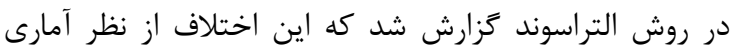

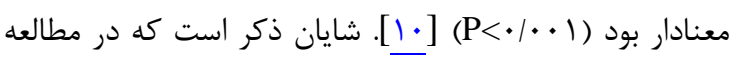

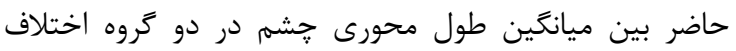

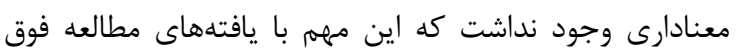

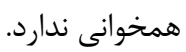

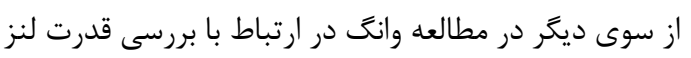

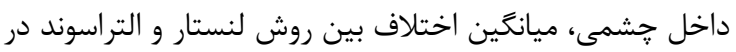

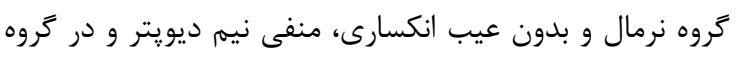

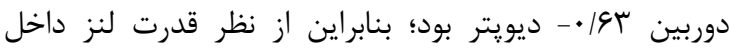

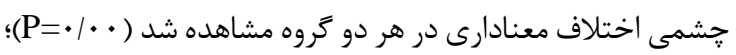

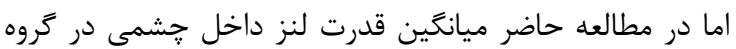

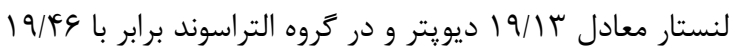

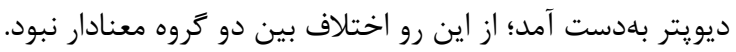

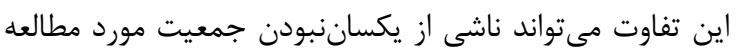

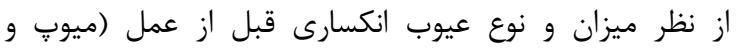

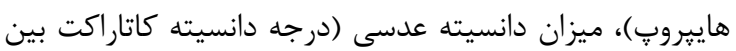

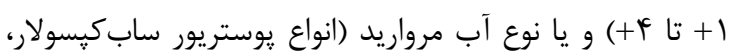

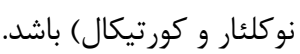

كام مههم در اصلاح ديد بيماران و رفع عيوب انكسارى قبل از

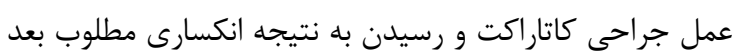

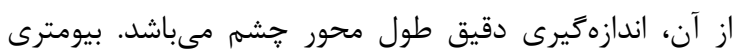

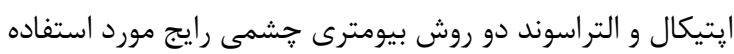

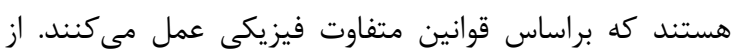
آنجايى كه براى انجام Partial Coherence Interferometry

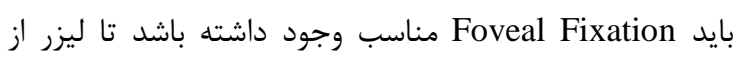

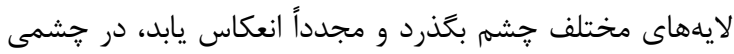

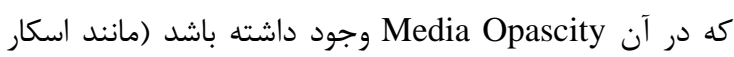

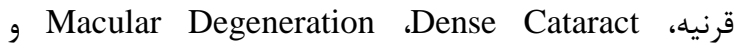
اكسنتريك فيكاسيون)، بيومترى به روش PCI كاربرد ندارد.

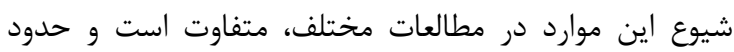

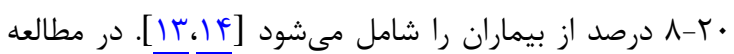

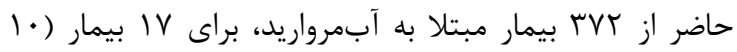

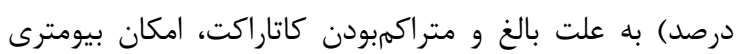

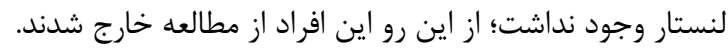

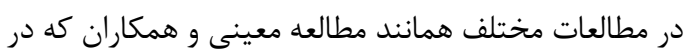

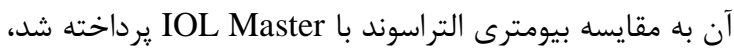

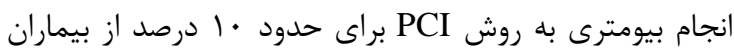

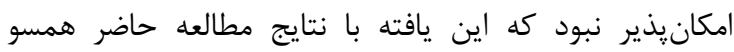

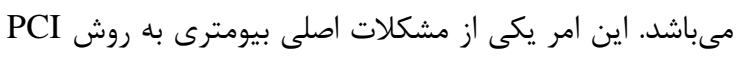

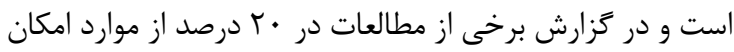

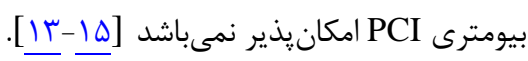

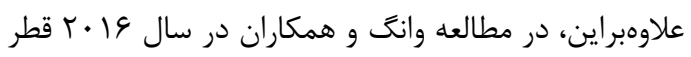
قدامى خلفى جشم در دو روش لنستار و و 
وجود ندارد؛ اما در • ا درصد از حجم نمونه گروه لنستار، انجام

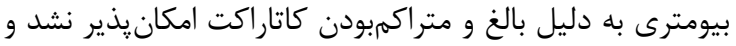

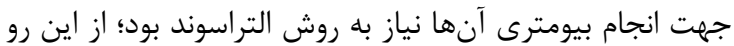

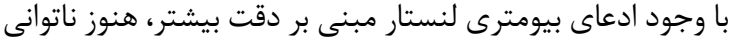

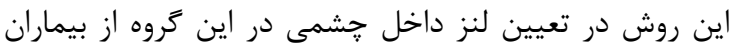

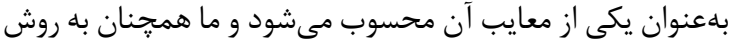
بيومترى التراسوند در شرايط خاص نيازمند هستيهم.

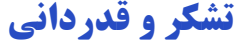

اين مقاله بركرفته از ياياننامه دكترى حرفهاى يزشكى دانى

مصوب دانشخاه علوم يزشكى همدان مئباشد. بدينوسيله

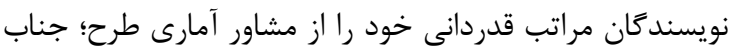

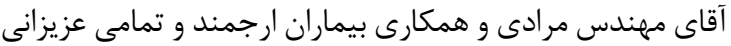

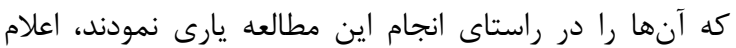

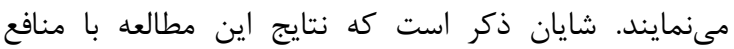
نويسندگان در تعارض نمىباشد.

\section{REFERENCES}

1. Hashemi H, Alipour F, Fotouhi A, Alaeddini F, Rezvan F, Mehravaran S, et al. Iranian cataract surgery survey, design and study protocol. Iran J Ophthalmol. 2010;22(2):39.

2. Fotouhi A, Hashemi H, Mohammad K, Jalali KH. The prevalence and causes of visual impairment in Tehran: the Tehran Eye Study. Br J Ophthalmol. 2004;88(6):740-5. PMID: 15148203

3. Bariah MA, Zainora M, Nazirin A. Comparison of refractive outcomes in post cataract surgery using measurements from immersion and contact A-scan biometry techniques. Malaysian J Health Sci. 2012;10(1):13-8.

4. Hoffer KJ, Hoffmann PC, Savini G. Comparison of a new optical biometer using swept-source optical coherence tomography and a biometer using optical low-coherence reflectometry. J Cataract Refract Surg. 2016;42(8):1165-72. PMID: 27531293 DOI: 10.1016/j.jcrs.2016.07.013

5. Falhar M, Rehak J. The contact and immersion Ultrasound methods compared using the ray tracing method. Optica Appl. 2010;40(1):77-92.

6. Uckmann MS, Stattin M, Zehetner C, Neururer S, Speicher L. Comparison of two optical biometric devices for intraocular lens calculation. Ophtalmologe. 2018;10(2):10718. PMID: 29372303 DOI: 10.1007/s00347-018-0655-7

7. Fontes BM, Fontes BM, Castro E. Intraocular lens power calculation by measuring axial length with partial optical coherence and ultrasonic biometry. Arq Bras Oftalmol. 2011;74(3):166-70. PMID: 21915441

8. Velez-Montoya R, Shusterman EM, López-Miranda MJ, Mayorquin-Ruiz M, Salcedo-Villanueva G, Quiroz-Mercado $\mathrm{H}$, et al. Comparison of the biometric values obtained by two different A-mode ultrasound devices (Eye Cubed vs. PalmScan): a transversal, descriptive, and comparative study. BMC Ophthalmol. 2010;10:18-24. PMID: 20334670 DOI: 10.1186/1471-2415-10-8

9. Jasvinder S, Khang TF, Sarinder KK, Loo VP, Subrayan V. Agreement analysis of LENSTAR with other techniques of biometry. Eye (Lond). 2011;25(6):717-24. PMID: 21394115 DOI: $10.1038 /$ eye.2011.28

10. Buckhurst PJ, Wolffsohn JS, Shah S, Naroo SA, Davies LN, Berrow EJ. A new optical low coherence reflectometry

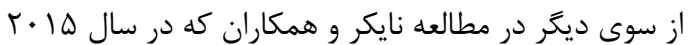

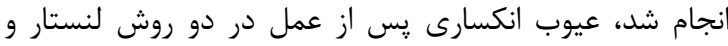

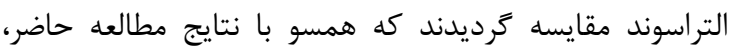
تفاوت معنادارى از نظر عيوب انكسارى يس از عمل در بين دو

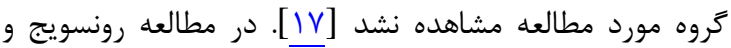

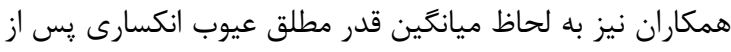
عمل تفاوت معنادارى بين دو روش بيومترى وجود نداشت [1/11] در مطالعه حاضر ميانگين قدر مطلق عيوب انكسارى يس از عمل

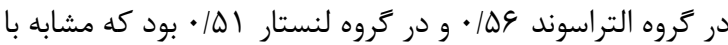

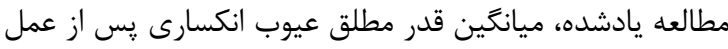
در دو گروه تفاوت معنادارى نداشت.

\section{نتيجه تَيرى}

بر مبناى نتايج مشخص شد كه در مقايسه دو روش بيومترى

التراسوند و لنستار در تعيين طول محور جشم و ميزان عيوب

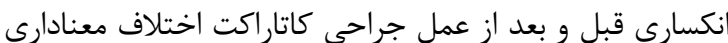

device for ocular biometryin cataract patients. $\mathrm{Br} J$ Ophthalmol. 2009;93(7):949-53. PMID: 19380310 DOI: 10.1136/bjo.2008.156554

11. Rohrer K, Frueh BE, Walti R, Clemetson IA, Tappeiner C, Goldblum D. Comparison and evaluation of ocular biometry using a newnoncontact optical low-coherence reflectometer. Ophthalmology. 2009;116(11):2087-92. PMID: 19744720 DOI: 10.1016/j.ophtha.2009.04.019

12. Riordan-Eva P, Whitcher JP. Vaughan \& Asbury's general ophthalmology. New York: McGraw-Hill; 2011.

13. Moeini H, Eslami F, Rismanchian A, Akhlaghi MR, Najafianjaz A. Comparison of ultrasound and optic biometry with respect to eye refractive errors after phacoemulsification. J Res Med Sci. 2008;13(2):43-7.

14. Rajan MS, Keilhorn I, Bell JA. Partial coherence laser interferometry vs conventional ultrasound biometry in intraocular lens power calculations. Eye (Lond). 2002;16(5):552-6. PMID: 12194067 DOI: 10.1038/sj.eye. 6700157

15. Raymond S, Favilla I, Santamaria L. Comparing ultrasound biometry with partial coherence interferometry for intraocular lens power calculations. Invest Ophthalmol Vis Sci. 2009;50(6):2547-52. PMID: 19168907 DOI: 10.1167/ iovs.08-3087

16. Wang XG, Dong J, Pu YL, Liu HJ, Wu Q. Comparison axial length measurements from three biometric instruments in high myopia. Int J Ophthalmol. 2016;9(6):876-80. PMID: 27366691 DOI: 10.18240/ijo.2016.06.15

17. Naicker P, Sundralingam S, Peyman M, Juana A, Mohamad NF, Win MM, et al. Refractive outcomes comparison between the Lenstar LS 900® optical biometry and immersion A-scan ultrasound. Int Ophthalmol. 2015;35(4):459-66. PMID: 25024102 DOI: 10.1007/s10792-014-9970-4

18. Rončević MB, Bušić M, Čima I, Elabjer BK, Bosnar D, Miletić D. Comparison of optical low-coherence reflectometry and applanation ultrasound biometry on intraocular lens power calculation. Graefe's Arch Clin Exp Ophthalmol. 2011;249(1):69-75. DOI: 10.1007/s00417-0101509-4 